\title{
gु
}

\section{Spin Transfer from an Optically Pumped Alkali Vapor to a Solid}

\author{
K. Ishikawa, * B. Patton, Y. -Y. Jau, and W. Happer \\ Joseph Henry Laboratory, Department of Physics, Princeton University, Princeton, New Jersey 08544, USA
}

(Received 12 January 2007; published 4 May 2007)

\begin{abstract}
We report enhancement of the spin polarization of ${ }^{133} \mathrm{Cs}$ nuclei in $\mathrm{CsH}$ salt by spin transfer from an optically pumped cesium vapor. The nuclear polarization was 4.0 times the equilibrium polarization at $9.4 \mathrm{~T}$ and $137^{\circ} \mathrm{C}$, with larger enhancements at lower fields. This work is the first demonstration of spin transfer from a polarized alkali vapor to the nuclei of a solid, opening up new possibilities for research in hyperpolarized materials.
\end{abstract}

DOI: $10.1103 /$ PhysRevLett.98.183004

PACS numbers: 32.80.Bx, 32.30.Dx, 33.40.+f, 76.60.-k

We describe a new method of polarizing nuclei in a solid by exposing it to an optically pumped alkali-metal vapor. The aim of this work is to develop a way to hyperpolarize nuclei which cannot be conveniently polarized by other methods [1-4]. In our initial experiments with cesium hydride films, we observed ${ }^{133} \mathrm{Cs}$ nuclear polarization enhancements of 4.0 over the equilibrium value at a magnetic field of $B=9.4 \mathrm{~T}$ and a temperature of $137^{\circ} \mathrm{C}$. These data are shown in Fig. 1. Higher enhancements have been measured in subsequent experiments conducted at 2.7 T. Much larger enhancements should be possible as we learn more about the detailed physics of this process.

The sample cells used in these experiments were cylindrical Pyrex cells, $13 \mathrm{~mm}$ in diameter and $38 \mathrm{~mm}$ in length, with optical-quality windows at both ends. These were attached to a glass manifold connected to a gas handling and vacuum pumping station. An ampoule of cesium (Chemetall $\mathrm{GmbH}$ ) with a break seal was also attached to the manifold. The cells were evacuated with a turbomolecular pump while being baked in an oven at $350{ }^{\circ} \mathrm{C}$ for several days until the residual pressure fell below $\sim 5 \times$ $10^{-8}$ Torr. The manifold was then cooled to room temperature and the break seal was shattered with a glassencapsulated magnetic hammer. Cesium was transferred into the cells by distillation with the flame of a gas torch, forming a thin alkali film on the inner walls of the cells. Hydrogen gas at a pressure of $\sim 700$ Torr was then added to the manifold. A smaller oven was placed around the cells and heated to $150{ }^{\circ} \mathrm{C}$ for several hours, sufficient time for most of the cesium metal on the cell walls to react with the $\mathrm{H}_{2}$ gas to form $\mathrm{CsH}$ salt. The remaining $\mathrm{H}_{2}$ was then evacuated and another thin film of Cs metal was distilled on top of the salt film and reacted with $\mathrm{H}_{2}$ gas as before. This layering process was repeated a total of four times to produce a film of $\mathrm{CsH}$ salt about $10^{-3} \mathrm{~cm}$ thick. In some cells the $\mathrm{CsH}$ had a slight blue coloration, possibly due to a thin layer of Cs metal on its surface or color centers within the salt crystals. The cells were evacuated one final time and a droplet of pure cesium metal was distilled into the stem of each to provide the alkali vapor needed for optical pumping. Finally each cell was filled with subatmospheric pressures of $\mathrm{N}_{2}$ quenching gas and flame-sealed to remove it from the manifold. The cells used in these experiments were filled with $\mathrm{N}_{2}$ pressures of $11,20,100,200$, and 700 Torr at $20^{\circ} \mathrm{C}$.

As sketched in Fig. 2, each sample cell was placed in an NMR coil contained within an oven which was inserted into a superconducting magnet (Oxford Instruments). The oven was electrically heated to a temperature of $130-170{ }^{\circ} \mathrm{C}$ [5] to provide an adequate atomic number density $[\mathrm{Cs}]$. Once the cell temperature reached equilibrium ( 2 to 3 hours later) the Cs vapor in the cells was optically pumped with $894 \mathrm{~nm}\left(6 S_{1 / 2} \rightarrow 6 P_{1 / 2}\right)$ light from a Coherent 899-29 Ti:sapphire ring laser. The Ti:sapphire output power was approximately $1 \mathrm{~W}$. The light was trans-

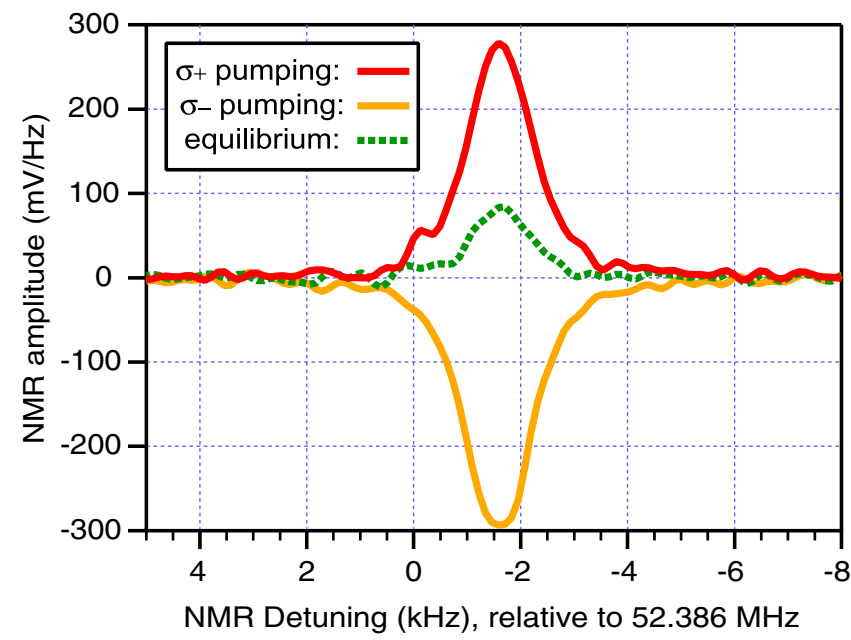

FIG. 1 (color online). NMR spectra of ${ }^{133} \mathrm{Cs}$ in $\mathrm{CsH}$ salt at 9.4 T. The cell contains a film of $\mathrm{CsH}$, a separate droplet of $\mathrm{Cs}$ metal to provide a vapor for optical pumping, and 11 Torr of $\mathrm{N}_{2}$ gas. The dashed trace is the NMR signal with no optical pumping; the red (positive) and orange (negative) traces show the hyperpolarized NMR signal obtained when the alkali vapor is pumped with $\sigma_{+}$and $\sigma_{-}$light, respectively, at the $6 S_{1 / 2} \rightarrow$ $6 P_{1 / 2}$ absorption line. Although the negative signal enhancement was larger than the positive enhancement in this case, the two were much more symmetric in other trials. 


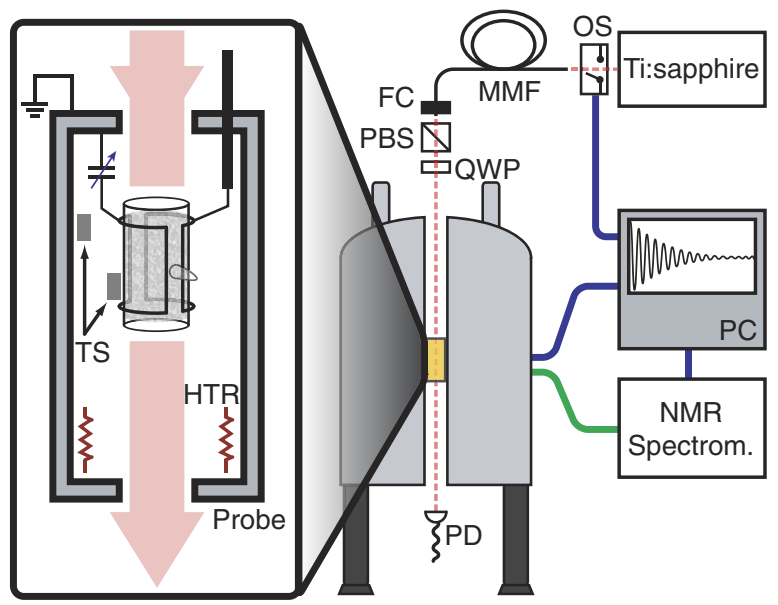

FIG. 2 (color online). The experimental apparatus. The Ti:sapphire laser light was routed through an optical shutter (OS) and into multimode fiber (MMF). At the output of the fiber coupler (FC) the light was polarized by the beam-splitter cube (PBS) and quarter-wave plate (QWP). A photodiode (PD) was used to verify optical absorption prior to the NMR experiment. Inside the NMR probe (inset), two temperature sensors (TS) were used to measure the cell and probe temperatures; the oven temperature was regulated by a resistive heater (HTR) controlled by the PC.

ported from the laser to the magnet through a multimode optical fiber (ThorLabs). A fiber coupler produced a collimated light beam directed down the axis of the magnet to the sample cell, uniformly illuminating the cell with a power density $P=50-150 \mathrm{~mW} \mathrm{~cm}^{-2}$. A polarizing beamsplitter cube and quarter-wave plate were placed at the output of the fiber to provide $\sigma_{+}$or $\sigma_{-}$circularly polarized light, as needed. The laser was tuned to the desired optical resonance frequency $\nu_{\text {op }}$, approximately $334940 \mathrm{GHz}$ for $\sigma_{-}$transitions and $335290 \mathrm{GHz}$ for $\sigma_{+}$transitions. (In contrast to most optical pumping experiments, these transitions have very different frequencies because of the large Zeeman splitting of electron levels in the $9.4 \mathrm{~T}$ magnetic field.) The laser frequency was continuously monitored with a WA-1150 wave meter (EXFO Inc.).

The ${ }^{133} \mathrm{Cs}$ nuclear magnetization was detected with a custom-made phase-sensitive nuclear magnetic resonance (NMR) spectrometer. Free induction decays (FIDs) following an excitation pulse were recorded on a Yokogawa DL708E oscilloscope and uploaded to a personal computer (PC) for data processing. Both the in-phase and quadrature transients were Fourier transformed, and the phases were adjusted to make the in-phase peak as symmetric as possible. This peak was fit to a Gaussian function and the area under the Gaussian fit was taken as a measure of the cesium nuclear magnetization. The NMR resonance of ${ }^{133} \mathrm{Cs}$ in $\mathrm{CsH}$ was observed at $52.39 \mathrm{MHz}$ while the frequency of pure cesium metal was measured to be $53.15 \mathrm{MHz}$, the difference being due to the Knight shift [6]. FIDs were obtained alternately from unpumped samples - in which the nuclear polarization had its equilibrium value - and from optically pumped samples where the magnitude of the polarization could be substantially larger.

To acquire an unpumped signal, the cell was left in the magnetic field for a time much longer than the ${ }^{133} \mathrm{Cs}$ nuclear relaxation time $T_{1}$ (approximately $170 \mathrm{~s}$ at $135^{\circ} \mathrm{C}$ ) and an FID was recorded. Then the optical shutter was opened and the laser light was allowed to pass through the cell. After a pumping time $T_{p}(180-400$ seconds), an FID signal was recorded again to obtain a pumped signal. The shutter was then closed and after a delay $T_{p}$ another unpumped signal was recorded; this averaging cycle was repeated until the signal-to-noise ratio allowed for a reliable determination of the signal enhancement. Data were acquired for both $\sigma_{+}$and $\sigma_{-}$pumping to eliminate systematic effects. For the two cells with low $\mathrm{N}_{2}$ pressures only a few such averages were necessary, since even a single NMR spectrum could reveal enhancement due to optical pumping. For the higher-pressure cells, many more averaging cycles were required (up to 140).

The transport of angular momentum to the walls can be understood with a simple model where the expectation value of the longitudinal electron spin, $\left\langle S_{z}\right\rangle$, and of the nuclear spin $\left\langle I_{z}\right\rangle$ (in units of $\hbar$ ) obey coupled diffusion equations [7]:

$$
\begin{gathered}
\frac{\Gamma_{\mathrm{op}}}{2}\left[1-2\left\langle S_{z}\right\rangle\right]-\Gamma_{\mathrm{sr}}\left\langle S_{z}\right\rangle-\left\langle\dot{I}_{z}\right\rangle_{\mathrm{hfs}}+D \nabla^{2}\left\langle S_{z}\right\rangle=0, \\
\left\langle\dot{I}_{z}\right\rangle_{\mathrm{hfs}}+D \nabla^{2}\left\langle I_{z}\right\rangle=0 .
\end{gathered}
$$

The mean optical pumping rate is $\Gamma_{\mathrm{op}}=\sigma_{\mathrm{op}} P / h \nu_{\mathrm{op}}$, where $h$ is Planck's constant. A representative cross section for $\sigma_{+}$light at $9.4 \mathrm{~T}$ and a nitrogen density $\left[\mathrm{N}_{2}\right]$ of $1 \mathrm{amg}$ is $\sigma_{\mathrm{op}}=3.5 \times 10^{-13} \mathrm{~cm}^{2}$. For an optical power density of $P=100 \mathrm{~mW} \mathrm{~cm}^{-2}$, this gives $\Gamma_{\mathrm{op}}=1.56 \times 10^{5} \mathrm{sec}^{-1}$. During a collision between a $\mathrm{Cs}$ atom and a nitrogen molecule, the spin-rotation interaction [8], $\gamma \mathbf{N} \cdot \mathbf{S}$, causes $\left\langle S_{z}\right\rangle$ to damp at the rate $\Gamma_{\mathrm{sr}}=\kappa_{\mathrm{sr}}\left[\mathrm{N}_{2}\right] \quad\left(\kappa_{\mathrm{sr}}=\right.$ $\left.820 \mathrm{sec}^{-1} \mathrm{amg}^{-1}\right)[9,10]$, and the hyperfine-shift interaction [11], $\delta A \mathbf{I} \cdot \mathbf{S}$, transfers angular momentum from $\left\langle S_{z}\right\rangle$ to $\left\langle I_{z}\right\rangle$ at the rate $\left\langle\dot{I}_{z}\right\rangle_{\mathrm{hfs}}=\Gamma_{\mathrm{hfs}}\left[\epsilon\left\langle S_{z}\right\rangle-\left\langle I_{z}\right\rangle\right]$, where $\Gamma_{\mathrm{hfs}}=$ $\eta_{I}^{2} \Gamma_{C} / 4=\kappa_{\mathrm{hfs}}\left[\mathrm{N}_{2}\right]\left(\kappa_{\mathrm{hfs}}=55 \mathrm{sec}^{-1} \mathrm{amg}^{-1}\right)$. The Carver rate $\Gamma_{C}$ is an isotope-independent measure of the relaxation due to hyperfine-shift collisions $[7,10,12]$. The isotope efficiency factor is $\eta_{I}=\mu_{I} / 2 I \mu_{N}$, where $\mu_{I}$ is the nuclear moment and $\mu_{N}$ is the nuclear magneton. The paramagnetic coefficient [2] is $\epsilon=2 I(I+1)-2\left\langle I_{z}^{2}\right\rangle$. The diffusion coefficient for Cs atoms in the nitrogen buffer gas is $D=D_{0} /\left[\mathrm{N}_{2}\right]$ with $D_{0}=0.087 \mathrm{~cm}^{2} \mathrm{sec}^{-1}$ amg [13].

The mean distance an unpolarized atom diffuses before absorbing a photon is $\lambda_{\mathrm{op}}=\sqrt{D / \Gamma_{\mathrm{op}}}$. For the representative case of $\left[\mathrm{N}_{2}\right]=1 \mathrm{amg}$, we would have $\lambda_{\text {op }}=7.5 \times$ $10^{-4} \mathrm{~cm}$. The solution [2] of (1) under these conditions is very nearly

$$
2\left\langle S_{z}\right\rangle=\frac{\Gamma_{\mathrm{op}}\left(1-e^{-\zeta / \lambda_{\mathrm{op}}}\right)}{\Gamma_{\mathrm{op}}+\Gamma_{\mathrm{sr}}+\Gamma_{\mathrm{hfs}}\left[\epsilon-2\left\langle I_{z}\right\rangle\right]},
$$


where $\zeta$ is the distance into the gas from the wall. Over most of the cell volume, $\left\langle S_{z}\right\rangle \approx 1 / 2$, but it drops to zero at the wall over a distance of order $\lambda_{\text {op }}$.

The current density of longitudinal electron spin to the wall is

$$
J_{S}=[\mathrm{Cs}] D \frac{\partial\left\langle S_{z}\right\rangle}{\partial \zeta}=\frac{[\mathrm{Cs}] \sqrt{\Gamma_{\mathrm{op}} D}}{2},
$$

where we used (3) to evaluate $\partial\left\langle S_{z}\right\rangle / \partial \zeta$ at $\zeta=0$, and where we have made use of the fact that $\Gamma_{\mathrm{op}} \gg \Gamma_{\mathrm{sr}}, \Gamma_{\mathrm{hfs}}$.

At the high magnetic fields of this experiment the energy basis states are very nearly products of $\left|S, m_{S}\right\rangle$ and $\left|I, m_{I}\right\rangle$, the eigenfunctions of $S_{z}$ and $I_{z}$. Equation (2) indicates that direct optical pumping of the cesium nucleus is negligible at the high fields of this experiment since absorption of a $\sigma_{+}$photon via an allowed transition increases the azimuthal quantum number $m_{J}$ from $m_{S}$ to $m_{S}+1$ without changing $m_{I}$. The large magnetic field nearly eliminates transfer of angular momentum between $\left\langle S_{z}\right\rangle$ and $\left\langle I_{z}\right\rangle$ during spin-exchange collisions between pairs of alkali-metal atoms [14]. For the allowed $\sigma_{+}$pumping modeled above, the nucleus is polarized as a result of hyperfine-shift collisions. Consider a "pancake cell" with parallel walls spaced a distance $2 a$ apart. Let $z$ be the distance from the midplane of the cell toward one of the walls. If we replace the paramagnetic coefficient $\epsilon$ by a constant mean value $\bar{\epsilon}$ the solution to (2) is

$$
\left\langle I_{z}\right\rangle=\frac{\bar{\epsilon}}{2}\left[1-\frac{\cosh \left(z / \lambda_{\mathrm{hfs}}\right)}{\cosh \left(a / \lambda_{\mathrm{hfs}}\right)}\right]
$$

with a diffusion length $\lambda_{\mathrm{hfs}}=\sqrt{D / \Gamma_{\mathrm{hfs}}}$. The current density of $\left\langle I_{z}\right\rangle$ into the wall is

$$
J_{I}=[\mathrm{Cs}] D \frac{\partial\left\langle I_{z}\right\rangle}{\partial z}=\frac{[\mathrm{Cs}] D \bar{\epsilon} \tanh \left(a / \lambda_{\mathrm{hfs}}\right)}{2 \lambda_{\mathrm{hfs}}} .
$$

In Fig. 3 we show the currents $J_{S}$ and $J_{I}$ as a function of the $\sigma_{+}$pumping light frequency, computed with a more detailed model that considers: (i) the population of each of the 16 energy sublevels of the ground-state Cs atom, (ii) the different optical pumping rates out of the different sublevels and their dependence on laser frequency and polarization, (iii) the admixtures to the uncoupled states $\left|S, m_{S}\right\rangle\left|I, m_{I}\right\rangle$ caused by the fact that the Zeeman splitting, while very large, is not infinitely large compared to the hyperfine interaction, and (iv) the cylindrical shape of the cells. The electron current $J_{S}$ is expected to reach a maximum when the laser is tuned to the allowed $\sigma_{+}$transition (a), where the results of the detailed calculation are nearly the same as those of the convenient, approximate expressions (4) and (6). In addition, there are interesting enhancements of the nuclear current $J_{I}$ when the laser is tuned to the forbidden transitions (b), (c), and (d), which occur because of the atomic basis states are not quite pure products $\left|S, m_{S}\right\rangle\left|I, m_{I}\right\rangle$. For these transitions, $\Delta m_{J}=0$ and $\Delta m_{I}=+(-) 1$ for $\sigma_{+(-)}$light. The electron spin

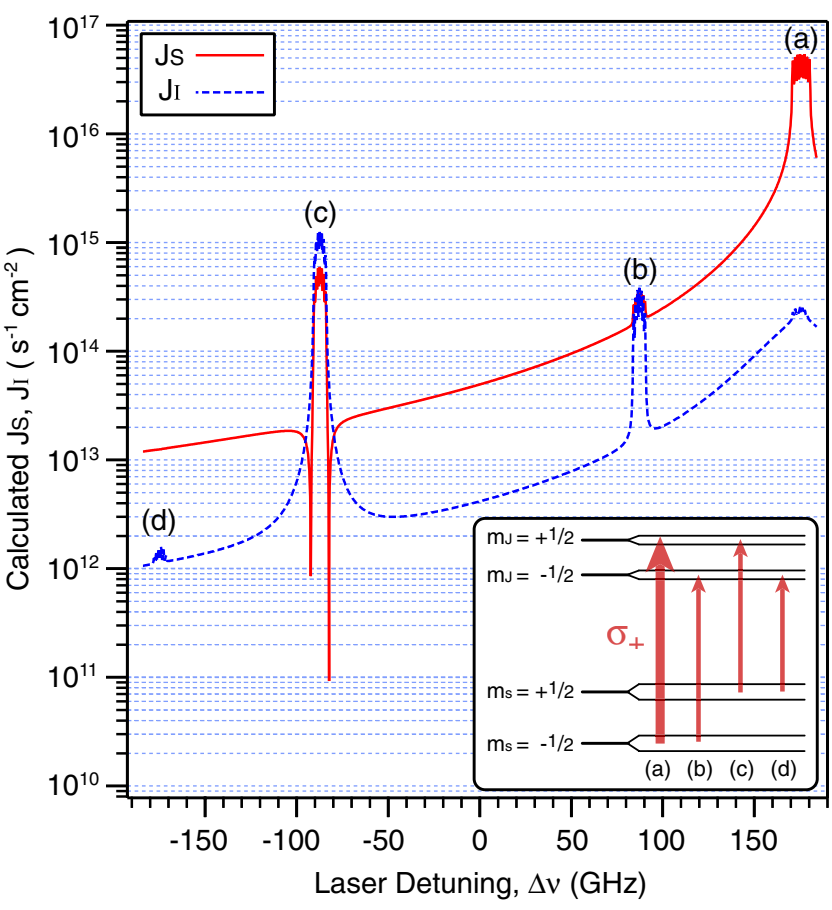

FIG. 3 (color online). Modeled diffusion currents $J_{S}$ and $J_{I}$ for a ${ }^{133} \mathrm{Cs}$ vapor at a temperature of $135^{\circ} \mathrm{C}$, with 11 Torr of nitrogen buffer gas in magnetic field $B=9.4 \mathrm{~T}$, pumped with $100 \mathrm{~mW} \mathrm{~cm}^{-2}$ of $\sigma_{+}$light. Note that $J_{S}$ is actually negative at resonance (c) at $-87.5 \mathrm{GHz}$; the absolute value has been taken to show its magnitude. Inset shows the allowed transition (a) and the forbidden transitions (b), (c), and (d) for $\sigma_{+}$light; these correspond to the resonances shown in the plot. Laser detuning is measured with respect to the $6 S_{1 / 2} \rightarrow 6 P_{1 / 2}$ transition at zero magnetic field.

flux at these transitions is a result of depopulation pumping of the ground-state electron energy levels and as such does not depend on the helicity of the light used. The transitions (b), (c), and (d) are much more prominent at lower magnetic fields where there is more hyperfine mixing of the states. In preliminary experiments where we have pumped forbidden transitions at $2.7 \mathrm{~T}$, we have shown strong NMR signal enhancements in $\mathrm{CsH}$ films under conditions where most of the spin must have been carried by $J_{I}$.

The predicted pressure dependence of the currents $J_{S}$ and $J_{I}$ has been of great interest to us in our first experiments. Model results for the allowed transition (a) at $9.4 \mathrm{~T}$ are shown in Fig. 4. Most of the angular momentum reaching the walls is carried by the electron current. $J_{S}$ is inversely proportional to the buffer-gas pressure $p$, as predicted by (4) since $D \propto 1 / p$, and also $\Gamma_{\text {op }} \propto 1 / p$ when $p$ exceeds a few tens of Torr, where the pressure broadening of the optical absorption lines exceeds the Doppler linewidth. The much smaller current of angular momentum carried by the nucleus is proportional to $p$ for pressures below a few tens of Torr, and it reaches a saturation value at higher pressures. This is consistent with the predictions of (6), since $\lambda_{\mathrm{hfs}} \propto 1 / p$. For pressures low 


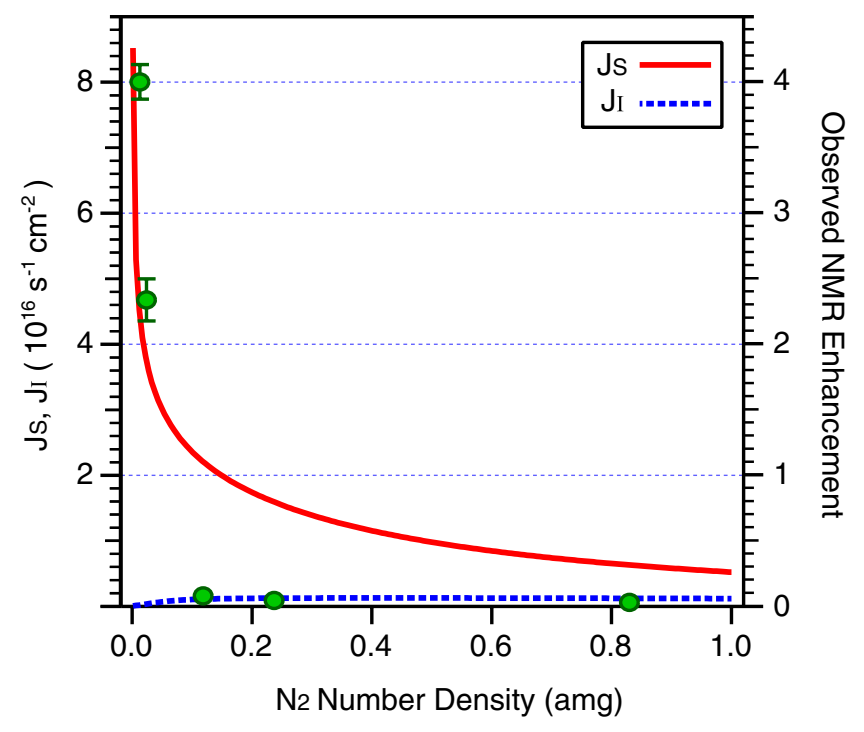

FIG. 4 (color online). Dependence of the measured ${ }^{133} \mathrm{Cs}$ NMR enhancement (points) on $\mathrm{N}_{2}$ number density. Also plotted are $J_{S}$ and $J_{I}$, the calculated electron and nuclear spin flux to the cell walls, for which uniform optical power density $p=$ $100 \mathrm{~mW} \mathrm{~cm}^{-2}$ and a temperature of $135^{\circ} \mathrm{C}$ were assumed. The $\mathrm{N}_{2}$ filling pressure has been converted to a number density in amagat, where $1 \mathrm{amg}$ is equal to the number density of an ideal gas at STP. Not all data points were taken at the same temperature; instead, the maximum enhancement for each cell is plotted. Radiation trapping was not considered in the numerical model.

enough that $a / \lambda_{\mathrm{hfs}} \ll 1$ we can write $\tanh \left(a / \lambda_{\mathrm{hfs}}\right)=$ $a / \lambda_{\mathrm{hfs}}$ and $J_{I}=[\mathrm{Cs}] \bar{\epsilon} a \Gamma_{\mathrm{hfs}} / 2 \propto p$. For pressures high enough that $\lambda_{\mathrm{hfs}} / a \ll 1$, we can write $\tanh \left(a / \lambda_{\mathrm{hfs}}\right) \approx 1$ and the current will saturate at the pressure-independent value, $J_{I}=[\mathrm{Cs}] I \sqrt{D \Gamma_{\mathrm{hfs}}}$, where we have set $\bar{\epsilon}=2 I$ corresponding to full polarization of the nuclear spin.

Also shown in Fig. 4 are the measured enhancements of ${ }^{133} \mathrm{Cs}$ NMR salt signals produced by pumping of the allowed resonance (a) for the five cells studied in this experiment. The enhancements should be directly proportional to the angular momentum currents into the salt surface and inversely proportional to the amount of $\mathrm{CsH}$ in each cell. Since the amount of $\mathrm{CsH}$ (and its surface area) was only approximately the same for all cells, one would hardly expect the observed signals to scale precisely with the modeled current densities. Nevertheless, the pressure dependence of the experimental signals closely resembles that modeled for $J_{S}$ and differs strongly from that modeled for $J_{I}$. This suggests that the NMR enhancements from optical pumping of allowed transitions at high fields are mostly due to the electron spin current. If the current is indeed carried by electrons it should be possible to polarize nuclei other than ${ }^{133} \mathrm{Cs}$ by placing solids containing these other nuclei in contact with spin polarized Cs or Rb vapors, which are particularly easy to optically pump. Nuclear cross relaxation might also allow the nuclear spin current $J_{I}$ of the vapor to polarize unlike nuclei on the surface as in SPINOE experiments [15], depending on the characteristic interaction time of vapor atoms with the surface. Especially interesting would be polarization transfer to ${ }^{6} \mathrm{Li}$ or ${ }^{7} \mathrm{Li}$ nuclei, which have much longer values of $T_{1}$ in many of their salts than does ${ }^{133} \mathrm{Cs}$ in $\mathrm{CsH}$. Such polarized salts could be of great use in spectroscopy and medical diagnostics.

We were unable to observe any enhancement of the metallic ${ }^{133} \mathrm{Cs}$ NMR signal in similar cells which contained no hydrogen. This is not surprising since $T_{1} \sim 10^{-3} \mathrm{~s}$ for nuclei in the metal (due to Korringa relaxation [16] ), about $10^{5}$ times shorter than for Cs nuclei in $\mathrm{CsH}$ salt.

In summary, we have shown that substantial amounts of angular momentum can be transferred to nuclei of solids in contact with optically pumped alkali-metal vapors. As future experiments reveal the details of this spin transfer and the polarization technique is optimized, larger NMR enhancements can be anticipated.

The authors would like to thank Mike Souza for his advice and glassblowing skills as well as Chemetall $\mathrm{GmbH}$ for high-purity Cs samples. This work was supported by the Air Force Office of Scientific Research.

*Present address: Graduate School of Material Science, University of Hyogo, Japan.

[1] B. M. Goodson, Annual Reports on NMR Spectroscopy (Elsevier Scientific, New York, 2005), Vol. 55, Chap. 6.

[2] T. Walker and W. Happer, Rev. Mod. Phys. 69, 629 (1997).

[3] S. T. Goertz, Nucl. Instrum. Methods Phys. Res., Sect. A 526, 28 (2004).

[4] J. Wolber, Nucl. Instrum. Methods Phys. Res., Sect. A 526, 173 (2004).

[5] A.N. Nesmeyanov, Vapor Pressure of the Elements (Academic, New York, 1963).

[6] C.H. Townes and W.D. Knight, Phys. Rev. 77, 852 (1950).

[7] D. K. Walter, W. M. Griffith, and W. Happer, Phys. Rev. Lett. 88, 093004 (2002).

[8] R. A. Bernheim, J. Chem. Phys. 36, 135 (1962).

[9] N. Beverini, P. Minguzzi, and F. Strumia, Phys. Rev. A 4, 550 (1971).

[10] A. Post, Ph.D. thesis, Princeton University, 2006.

[11] M. Arditi and T. R. Carver, Phys. Rev. 124, 800 (1961).

[12] Y.-Y. Jau, Ph.D. thesis, Princeton University, 2005.

[13] N. Beverini, Phys. Rev. A 5, 993 (1972).

[14] T. Walker and L. W. Anderson, Nucl. Instrum. Methods Phys. Res., Sect. A 334, 313 (1993).

[15] T. Rõõm, S. Appelt, R. Seydoux, E. L. Hahn, and A. Pines, Phys. Rev. B 55, 11604 (1997).

[16] J. Korringa, Physica (Amsterdam) 16, 601 (1950). 